\title{
On Fuzzy Qualitative and Quantitative Softgoal Interdependency Graph
}

\author{
Md. Barique Quamar \\ M. Tech. Scholar, \\ Department of Computer Science and Engineering \\ Faculty of Engineering and Technology \\ Al-Falah University, Dhauj, \\ Faridabad, Haryana, India
}

\author{
Yahiya Gazi \\ M. Tech. Scholar, \\ Department of Computer Science and Engineering \\ Faculty of Engineering and Technology \\ Al-Falah University, Dhauj, \\ Faridabad, Haryana, India
}

\begin{abstract}
Non-Functional requirements serve as criteria for the selection and prioritization of software requirements. The objective of this paper is to propose an efficient method for the prioritization of non functional requirements. In this paper we use $\mathrm{L}^{-1}, \mathrm{R}^{-1}$ inverse function arithmetic principle and graded mean integration for the elicitation of decision maker's weight for the qualitative and quantitative softgoal interdependency graph to model the non-functional requirements. Finally the utilization of proposed method is demonstrated with the help of an example.
\end{abstract}

\section{Keyword}

Softgoal Interdependency graph (SIG), Non-functional requirements (NFR), Trapezoidal fuzzy numbers (TFN), FQQSIG.

\section{INTRODUCTION}

Research in the area of trustworthy software system has received much attention by software engineering community in recent year. In 2014 "Make in India" project was started to increase the number of software product start-up in the country. India software industry is expected to grow to $\$ 225$ billion by 2020.A software system can only be suitable if it is Trustworthy. Chen et. al., ${ }^{[4]}$ define software trustworthiness as a system which have the following characteristic like safety, security, reliability, maintainability etc. Software requirements are classified into functional requirements (FR) and non-functional requirements (NFR). Software system that lack FR i.e. Based on our literature review, we have identify different sets $[9,12,13,22,21,1]$ and NFR framework [9] and softgoal Interdependency graph (SIG) [8, 7, 5]. SIG mainly emphasis the importance of qualitative analysis, of NFRs. For example, Zayaraz and Sadana [2, 21], proposed a quantitative model that focus on priorities and analyzes the conflict NFRs. Marew [20], presented "quantified softgoal interdependency graph". In this graph precise values are used to express the degree of satisfaction. This method also used for arithmetic operation to calculate the contribution of NFRs. In 2011, Zhu [15], proposed a NFR tradeoff model in trustworthy software which uses a fuzzy qualitative and quantitative softgoal interdependency graph (FQQSIG). In this method RAGE defuzzification procedure is used to finish the defuzzification transformation process. In this paper, we proposed a fuzzy based approach for the non-functional prioritization using $\mathrm{L}^{-1}, \mathrm{R}^{-1}$ function arithmetic principle and graded mean integration representation.
This paper is organized as follows: Section II presents the proposed method section III we present a case study of proposed method and finally conclusion and future work.

what the system is supposed to do and NFRs i.e. how the system is suppose to behave, such as reliability and performance carry the risk of failing to meet the customer and user expectation [23, 3]. In literature, NFRs has received less attention throughout the eliciting, capturing, modeling, testing, and maintaining process of software requirements.

\section{PROPOSED METHOD}

In this section we present the proposed method a fuzzy based approach for the NFR tradeoff model in trustworthy software. Proposed method includes the following steps:

\section{Step 1: Identification of stakeholder}

Stakeholder identification is a most important activity of a requirements elicitation process. Therefore, the first step of our method is to identify the primary and secondary stakeholders $[17,18]$. Primary stakeholders include those who are central to any project initiative, i.e., beneficiaries, financial, politicians, sponsors, and decision maker. Secondary stakeholders include developers, experts, operators etc [19].

\section{Step 2: In this step we decompose the NFRs.}

Software requirements are divided into two categories: functional and non-functional [6]. Many researchers, including Boehm [26], Sommerville [25], and Chung [9], present various NFR classifications. These classifications contain the need for different application areas in different conditions. They are helpful for developers to quickly obtain the area knowledge and easily modify software requirements. In 2015 [16] there is another classification of NFR which is based on commonly used NFR's, on the basis of definition and attributes of NFRs, and on the basis of conflicted NFR's. In "commonly used NFR", only those NFRs are considered which are useful for the different types of systems like information system, web based system etc. On the basis of literature review, only five types of NFRs that are commonly used in different types of systems [14] like Performance, Reliability, Usability, Security, and Maintainability. In second type of NFRs which is "on the basis of definition and attribute" is to identify those requirements which are clearly defined in the literature, like. Performance, Security Usability etc. and also identify those requirements which are supported by their attributes like Accessibility, Adaptability, Availability, Efficiency etc.[16]. This classification is further divided on the basis of the following: (i) Have Definition and Attributes (ii) Have Definition (iii) Without Definition and 
Attributes. The third type of classification of NFR is conflicted NFR for example, Accuracy and Performance ${ }^{[14}$, 16]. Conflicted NFRs further classify into three parts i.e. Absolute conflicted NFRs, Relative Conflicted NFRs and NFRs that are Never Conflicted. There are some NFRs which may conflict with other NFRs [16]. For example, reliability and performance.

Step 3: In this step we collect expert fuzzy assessment.

Step 4: To elicit the DMs weight vectors we use the $\mathbf{L}^{-1}-\mathbf{R}^{-1}$ inverse function arithmetic principle and graded mean integration representation [27].
Step 5: In this step we construct the FQQSIG graph.

\section{CASE STUDY}

In this section we applied the proposed method with help of five decision makers (DMs) opinion on NFR degree assessment of criteria. In this case study, we use seven ranking parameters i.e. Weak (W), Medium Weak (MW), Fair (F), Medium Strong(MS), Strong (S), Very Strong (VS), Extremely Strong (ES) etc. DMs fuzzy assessment parameter is shown in Table 1.

Table 1. Trapezoidal Fuzzy Linguistic variables set for NFR.

\begin{tabular}{|l|l|l|}
\hline Linguistic variables Set & Abbreviation & $\begin{array}{l}\text { Trapezoidal fuzzy numbers } \\
\text { S1(n1,n2,n3,n4)(i=1,.....,9) }\end{array}$ \\
\hline S1:Weak & W & $(0.176,0.235,0.294,0.353)$ \\
\hline S2:Medium Weak & MW & $(0.294,0.353,0.412,0.471)$ \\
\hline S3:Fair & F & $(0.412,0.471,0.529,0.588)$ \\
\hline S4:Medium Strong & MS & $(0.529,0.588,0.647,0.706)$ \\
\hline S5:Strong & S & $(0.647,0.706,0.765,0.824)$ \\
\hline S6:Very Strong & VS & $(0.765,0.824,0.882,0941)$ \\
\hline S7:Extremly Strong & ES & $(0.882,0.941,1,1)$ \\
\hline
\end{tabular}

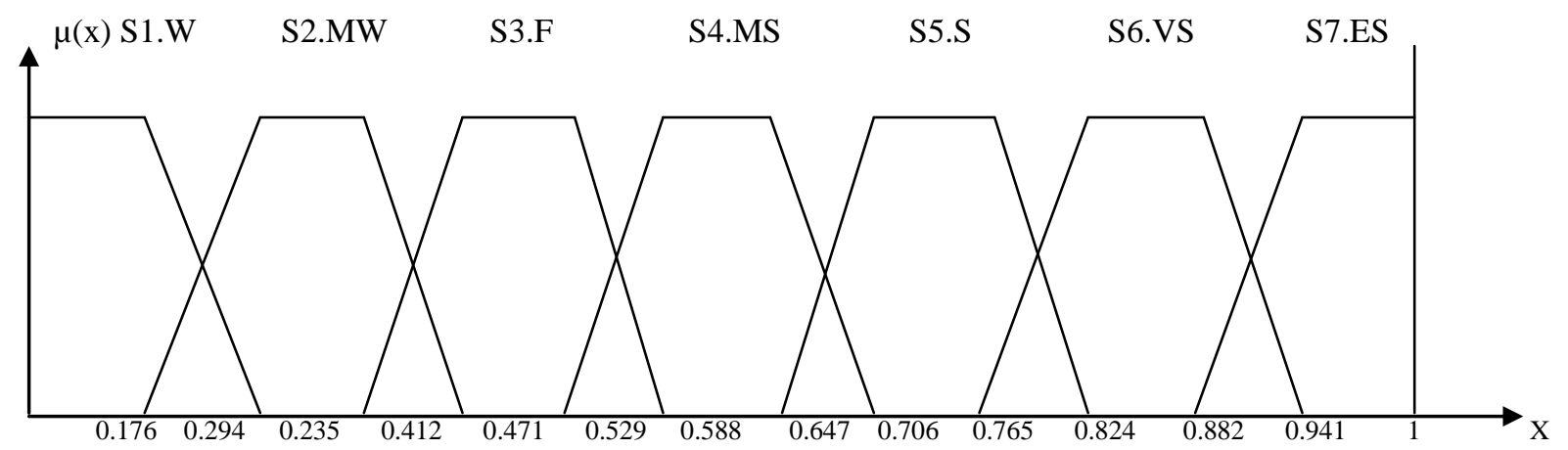

Fig. 2. Membership functions for a fuzzy set including seven variables.

Step 3: Collect expert fuzzy assessments

In this step to achieve TS system we decompose the Information system and proposed a NFR classification in [16].
We classify NFRs into three layers L1 ( $\mathrm{i}=1,2,3 \ldots \mathrm{n})$ in Table 2.

Table 2 Classification of NFRs and their fuzzy assessment by DMs

\begin{tabular}{|l|c|c|c|c|c|c|}
\hline NFRs(i=40) & DM1 & DM2 & DM3 & DM4 & DM5 & W i-j \\
& & & & & \\
\hline L1- Trustiness & F & W & MW & VW & F & 3.588 \\
\hline L2- IS/safety & F & VS & ES & S & VS & 3.901 \\
\hline L3- IS/Real & MS & S & F & MS & F & 2.969 \\
\hline L4- IS/web & S & MS & S & VS & MS & 3.322 \\
\hline L5- IS/Real/Web & & & F & MS & ES & 3.772 \\
\hline & & & & & \\
Secondary Layer & S & MS & F & VS & & \\
\hline L1-1 Provability & VS & S & S & MS & VS & 3.793 \\
\hline L1-2 Reusability & MS & VS & F & S & S & 3.44 \\
\hline L1-3 Standardizability & VS & MW & MS & F & VS & 3.205 \\
\hline L1-4 Traceability & & &
\end{tabular}




\begin{tabular}{|c|c|c|c|c|c|c|}
\hline L1-5 Viability & MW & $\mathrm{F}$ & $\mathrm{W}$ & MS & $\mathrm{F}$ & 2.269 \\
\hline \multicolumn{7}{|l|}{ Tertiary Layer } \\
\hline L2-1 Reliability & $\mathrm{S}$ & MS & ES & $\mathrm{S}$ & ES & 3.218 \\
\hline L2-2 Availability & VS & MW & $\mathrm{F}$ & ES & $\mathrm{S}$ & 3.431 \\
\hline Accuracy & $\mathrm{VS}$ & MS & MS & $\mathrm{S}$ & $\mathrm{F}$ & 3.923 \\
\hline L2-4 Confidentiality & $\mathrm{S}$ & $\mathrm{S}$ & $\mathrm{F}$ & VS & ES & 3.783 \\
\hline L2-5 $\quad$ Verifiability & $\mathrm{F}$ & MS & MS & $\mathrm{S}$ & $\mathrm{F}$ & 2.969 \\
\hline L3-1 Interoperability & MS & $\mathrm{W}$ & $\mathrm{F}$ & $\mathrm{F}$ & MS & 2.504 \\
\hline L3-2 Privacy & $\mathrm{ES}$ & $\mathrm{S}$ & MS & $\mathrm{VS}$ & ES & 4.125 \\
\hline L3-3 Security & $\mathrm{S}$ & VS & ES & $\mathrm{F}$ & MS & 3.665 \\
\hline L3-4 Performance & $\mathrm{VS}$ & ES & $\mathrm{S}$ & MS & $\mathrm{F}$ & 3.665 \\
\hline L3-5 $\quad$ Usability & $\mathrm{F}$ & MS & $\mathrm{S}$ & MS & $\mathrm{F}$ & 2.969 \\
\hline L3-6 Completeness & $\mathrm{S}$ & $\mathrm{S}$ & $\mathrm{VS}$ & $\mathrm{F}$ & $S$ & 2.823 \\
\hline L3-7 Maturity & $\mathrm{F}$ & $\mathrm{F}$ & MS & MW & $\mathrm{W}$ & 3.058 \\
\hline L3-8 Compliance & MW & MW & $\mathrm{F}$ & MS & MW & 1.909 \\
\hline L3-9 Confidentiality & ES & $\mathrm{S}$ & VS & MS & $\mathrm{S}$ & 1.881 \\
\hline L3-10 Availability & $\mathrm{S}$ & MS & ES & $\mathrm{S}$ & $\mathrm{S}$ & 3.165 \\
\hline L3-11 Access control & $\mathrm{S}$ & $\mathrm{S}$ & $\mathrm{F}$ & MS & VS & 3.047 \\
\hline L3-12 Authentication & VS & $\mathrm{F}$ & ES & $\mathrm{S}$ & MS & 2.587 \\
\hline L3-13 Integrity & $\mathrm{S}$ & $\mathrm{F}$ & $\mathrm{F}$ & VS & MS & 3.048 \\
\hline L3-14 Learnability & MW & MS & MW & $\mathrm{S}$ & $\mathrm{F}$ & 2.586 \\
\hline L4-1 Operability & $\mathrm{W}$ & $\mathrm{S}$ & $\mathrm{F}$ & MS & MS & 3.047 \\
\hline L4-2 Ease of use & $\mathrm{S}$ & $\mathrm{F}$ & $\mathrm{S}$ & MS & MS & 2.588 \\
\hline L4-3 Usefulness & $\mathrm{W}$ & VS & MS & $\mathrm{F}$ & $\mathrm{F}$ & 2.116 \\
\hline L4-4 Productivity & $\mathrm{F}$ & ES & ES & $\mathrm{S}$ & VS & 2.112 \\
\hline L4-5 $\quad$ Response time & $\mathrm{VS}$ & $\mathrm{S}$ & $\mathrm{VS}$ & $\mathrm{F}$ & ES & 2.823 \\
\hline $\begin{array}{ll}\text { L4-6 } & \text { Space } \\
\end{array}$ & $\mathrm{S}$ & MS & $\mathrm{VS}$ & $\mathrm{F}$ & ES & 2.241 \\
\hline L4-7 Capacity & MS & $\mathrm{F}$ & $\mathrm{S}$ & $S$ & $\mathrm{~S}$ & 3.155 \\
\hline $\begin{array}{ll}\text { L4-9 } & \text { Throughput } \\
\end{array}$ & $\mathrm{VS}$ & $S$ & $\mathrm{~F}$ & $\mathrm{~S}$ & $\mathrm{VS}$ & 3.164 \\
\hline L4-10 Protected & ES & $\mathrm{F}$ & $\mathrm{S}$ & MS & VS & 3.664 \\
\hline L4-11 Genuine & $\mathrm{S}$ & MS & $\mathrm{F}$ & $\mathrm{F}$ & $\mathrm{S}$ & 3.087 \\
\hline L4-12 Bonafide & $\mathrm{F}$ & MS & $\mathrm{S}$ & $\mathrm{F}$ & $\mathrm{S}$ & 3.085 \\
\hline
\end{tabular}

Step 4: Apply $\mathrm{L}^{-1}, \mathrm{R}^{-1}$ inverse function arithmetic principle and graded mean integration for the elicitation decision maker's weight

In this paper we apply $L^{-1}, R^{-1}$ function arithmetic principal and graded mean method for the elicitation of decision maker's weight. A brief introduction is given below:

Let $A_{1}=\left(a_{1}, b_{1}, c_{1}\right)$ and $A_{2}=\left(a_{2}, b_{2}, c_{2}\right)$ be two trapezoidal fuzzy numbers as Fig. 1. The addition of $A_{1}$ and $A_{2}$ at h-level is:

$A_{1(h)} \oplus A_{2(h)}=\left(L_{A 1(h)}^{-1}+L_{A 2(h)}^{-1}, L_{A 1(h)}^{-1}+R_{A 2(h)}^{-1}, R_{A 1(h)}^{-1}+\right.$ $\left.L_{A 2(h)}^{-1}, R_{A 1(h)}^{-1}+R_{A 2(h)}^{-1}\right)$

$L_{A 1}$ and $R_{A 1}$ are the functions $\mathrm{L}$ and $\mathrm{R}$ of fuzzy number $\mathrm{A}_{1}$, respectively. $L_{A 1(h)}^{-1}$ and $R_{A 1(h)}^{-1}$ are the inverse functions of functions $L_{A 1}$ and $R_{A 1}$ at h-level, respectively. $L_{A 2}$ and $R_{A 2}$ are the functions $\mathrm{L}$ and $\mathrm{R}$ of fuzzy number $\mathrm{A}_{2}$, respectively. $L_{A 2(h)}^{-1}$ and $R_{A 2(h)}^{-1}$ are the inverse functions of functions $L_{A 2}$ and $R_{A 2}$ at h-level, respectively.

Suppose the membership functions of $A_{1}=\left(a_{1}, b_{1}, c_{1}\right)$ is

$f_{A 1}(x)=\left\{\begin{array}{ll}\frac{\left(x-a_{1}\right)}{\left(b_{1}-a_{1}\right)}, a_{1} \leq x \leq b_{1}, \\ \frac{\left(x-c_{1}\right)}{\left(b_{1}-c_{1}\right)}, b_{1} \leq x \leq c_{1} \\ 0, \quad \text { otherwise. }\end{array}\right\}$

Since
$L_{A 1}(x)=\frac{\left(x-a_{1}\right)}{\left(b_{1}-a_{1}\right)}, a_{1} \leq x \leq b_{1}$

$R_{A 1}(x)=\frac{\left(x-c_{1}\right)}{\left(b_{1}-c_{1}\right)}, b_{1} \leq x \leq c_{1}$

and

$$
\begin{array}{ll}
L_{A 1(h)}^{-1}=b_{1}+\left(b_{1}-a_{1}\right) h, & 0 \leq h \leq 1, \\
R_{A 1(h)}^{-1}=c_{1}+\left(b_{1}-c_{1}\right) h, & 0 \leq h \leq 1,
\end{array}
$$

Similarly, suppose the membership function of $A_{2}=\left(a_{2}, b_{2}, c_{2}\right)$ is

$f_{A 2}(x)=\left\{\begin{array}{cc}\frac{\left(x-a_{2}\right)}{\left(b_{2}-a_{2}\right)}, & a_{2} \leq x \leq b_{2}, \\ \frac{\left(x-c_{2}\right)}{\left(b_{2}-c_{2}\right)}, & b_{2} \leq x \leq c_{2}, \\ 0, & \text { otherwise. }\end{array}\right\}$

Since

$L_{A 2}(x)=\frac{\left(x-a_{2}\right)}{\left(b_{2}-a_{2}\right)}, \quad a_{2} \leq x \leq$
$b_{2}$,

$R_{A 2}(x)=\frac{\left(x-c_{2}\right)}{\left(b_{2}-c_{2}\right)}, \quad b_{2} \leq x \leq$

$c_{2}$, 
and

$$
\begin{gathered}
L_{A 2(h)}^{-1}=a_{2}+\left(b_{2}-a_{2}\right) h, \quad 0 \leq h \leq 1, \\
R_{A 2(h)}^{-1}=c_{2}+\left(b_{2}-c_{2}\right) h, \quad 0 \leq h \leq 1, \\
A_{1(h)} \oplus A_{2(h)}=\left(\begin{array}{l}
L_{A 1(h)}^{-1}+L_{A 2(h)}^{-1}, L_{A 1(h)}^{-1}+R_{A 2(h)}^{-1}, R_{A 1(h)}^{-1} \\
\left.+L_{A 2(h)}^{-1}, R_{A 1(h)}^{-1}+R_{A 2(h)}^{-1}\right)
\end{array}\right. \\
=\left[\left(a_{1}+\left(b_{1}-a_{1}\right) h\right)+\left(a_{2}+\left(b_{2}-a_{2}\right) h\right),\left(a_{1}+\right.\right. \\
b 1-a 1 h+c 2+b 2-c 2 h, c 1+b 1-c 1 h+a 2+b 2-a 2 h, \\
c 1+b 1-c 1 h+c 2+b 2-c 2 h .
\end{gathered}
$$

According to equation 1, we have that

Second, we introduce briefly the graded integration representation method. Chen and Hsieh proposed the graded integration representation method of fuzzy numbers $[10,11$, 12] based on the integral value of graded mean h-level of generalized fuzzy number. Here, we describe the meaning as follows.

In general, a generalized fuzzy number A is described as any fuzzy subset of the real line $R$, whose membership function $u_{A}$ satisfies the following conditions.

(1). $\boldsymbol{u}_{\boldsymbol{A}}(\boldsymbol{x})=\mathbf{0},-\infty<\boldsymbol{x} \leq \boldsymbol{c}$;

(2). $u_{A}(x)$ is strictly increasing on $[c, a]$;

(3). $u_{A}(x)=w, a \leq x \leq b$, where $0<w \leq 1$

(4). $u_{A}(x)$ is strictly increasing on $[b, d]$

(5). $\boldsymbol{u}_{\boldsymbol{A}}(\boldsymbol{x})=\mathbf{0}, \boldsymbol{d} \leq \boldsymbol{x} \leq \infty$;

(6). $u_{A}$ is a continuous mapping from $\mathbf{R}$ to the closed $[0,1]$

Here $\mathrm{a}, \mathrm{b}, \mathrm{c}$, and $\mathrm{d}$ are real numbers. We denote generalized fuzzy number A in Fig .1 as $\left(\mathrm{a}, \mathrm{b}, \mathrm{c}, \mathrm{d} ; \boldsymbol{W}_{\boldsymbol{A}}\right)_{\mathrm{LR}}$. When $\boldsymbol{W}_{\boldsymbol{A}}=\mathbf{1}$. we simplify notation as $\mathrm{A}=(\mathrm{a}, \mathrm{b}, \mathrm{c}, \mathrm{d})_{\mathrm{LR}}$

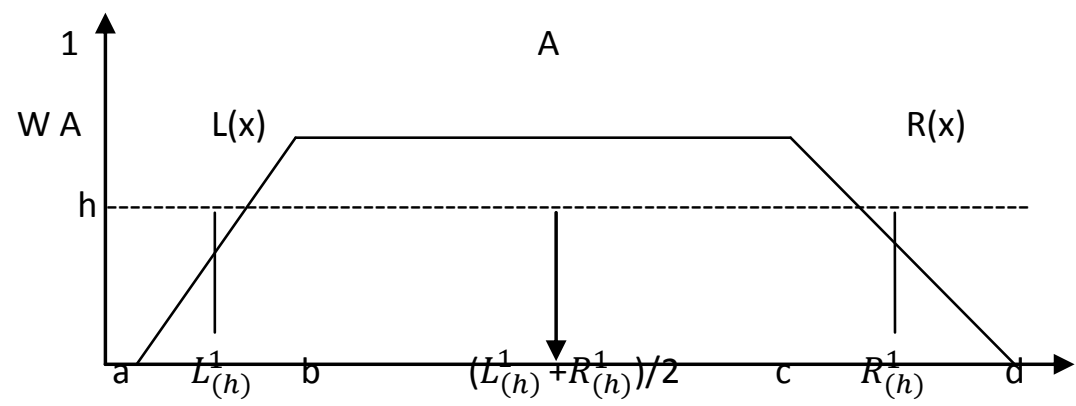

Fig 1.The graded mean h-level of fuzzy number $A=(a, b, c, d \text {; WA })_{L R}$.

Let $L^{1}$ and $R^{1}$ be the inverse function of the functions $L$ and $\mathrm{R}$,respectively ; then the graded mean h-level value of generalized number $\mathrm{A}$ is $\mathrm{h}\left(L_{(h)}^{1}+R_{(h)}^{1}\right) / 2$ as shown in figure. Then the graded mean integration representation of $\mathrm{A}$ is

$P(A)=\frac{\int_{0}^{w A}\left(h\left(L_{(h)}^{-1}+L_{(h)}^{-1}\right) / 2\right) d h}{\int_{0}^{w A} h d h}$

$$
\begin{aligned}
& =\frac{P(A)}{\int_{0}^{w A}(h(a+(b-a) h+d+(c-d) h) / 2) d h} \\
& =\frac{1}{6}(a+2 b+2 c+d)
\end{aligned}
$$

Here, a, b, c and d are numbers. We denote generalized fuzzy number $A$ in Fig. 2 as $A=(c, a, b, d)_{L R}$. When $w_{A}=1$, we simplify the notation as $\mathrm{A}=(\mathrm{a}, \mathrm{b}, \mathrm{c}, \mathrm{d})_{\mathrm{LR}}$.

Generalized triangular fuzzy number $\mathrm{K}=(\mathrm{a}, \mathrm{b}, \mathrm{c}, \mathrm{d})$ is a special case of generalized trapezoidal fuzzy number. The graded mean integration representation of the triangular fuzzy number $\mathrm{Y}$ becomes

$$
\begin{aligned}
& \operatorname{TFN}(K) \\
& =\frac{1}{6}(a+2 b+2 c \\
& +d)
\end{aligned}
$$

\section{Step 5: Construct FQQSIG graph}

In this step we construct FQQSIG graph .FQQSIG is an enhanced version of QSIG .In this step we assigned fuzzy linguistic variable i.e. we take a module of fees slip in this module we decomposes the NFRs through the prioritization till the last highest value by using fuzzy $\mathrm{L}^{-1}-\mathrm{R}^{-1}$ inverse linguistic variables function as shown in the Fig 4 the last attributes is completeness.After applying equation, we have identify the weights of different measuring parameters ,i.e. we take an example performance $\mathrm{VS}=0.853, \mathrm{ES}=0.960, \mathrm{~S}=0.735, \mathrm{MS}=0.617, \mathrm{~F}=0.5$ the final output in the figure is after normalized form. The normalized values (Fig. 4) are acquired by dividing the sum of all attributes values in the same layer; for example: $0.14=2.82 /(4.12+3.66+3.08+3.08+3.05+2.82)$. 


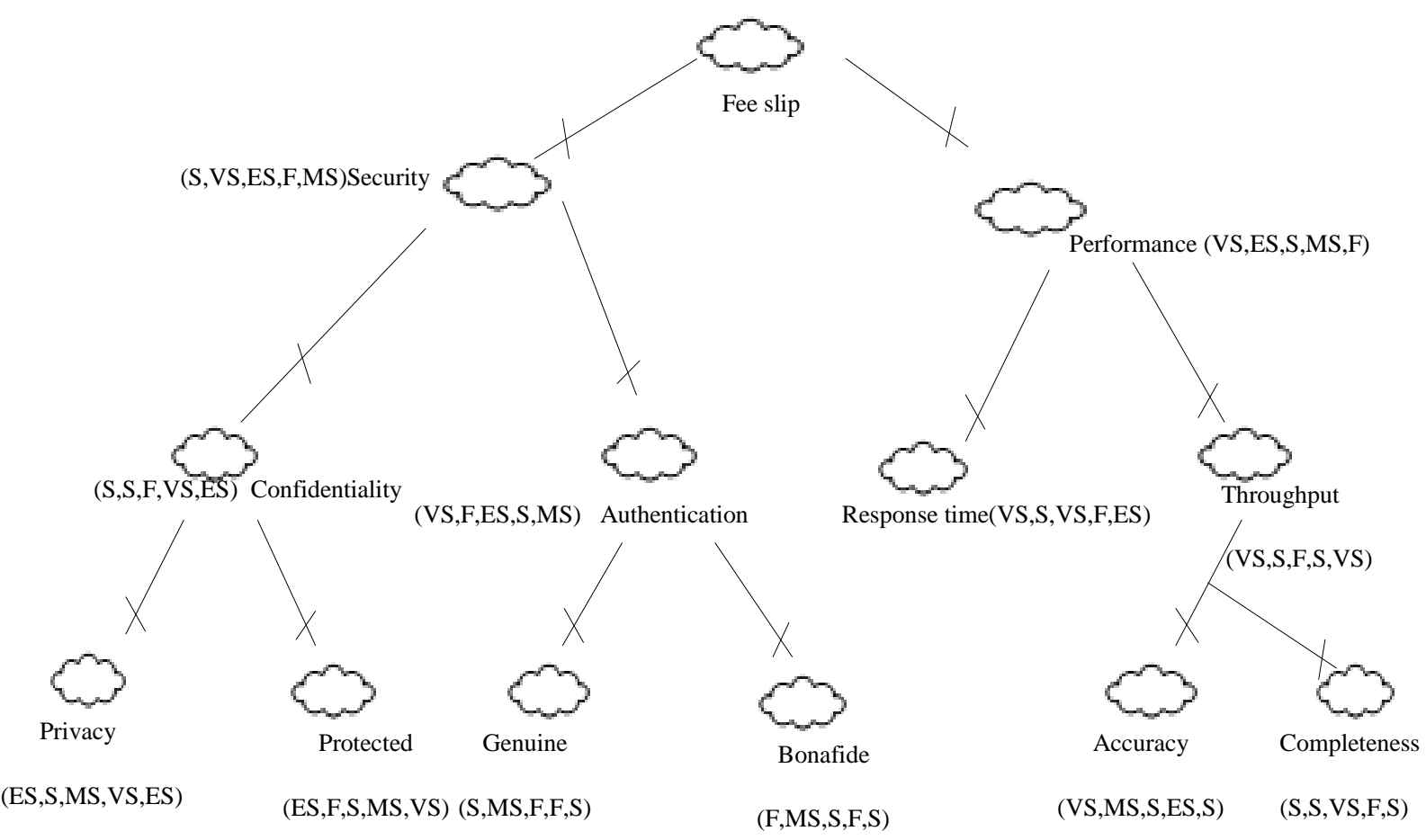

Fig 3.1.EFQQSIG

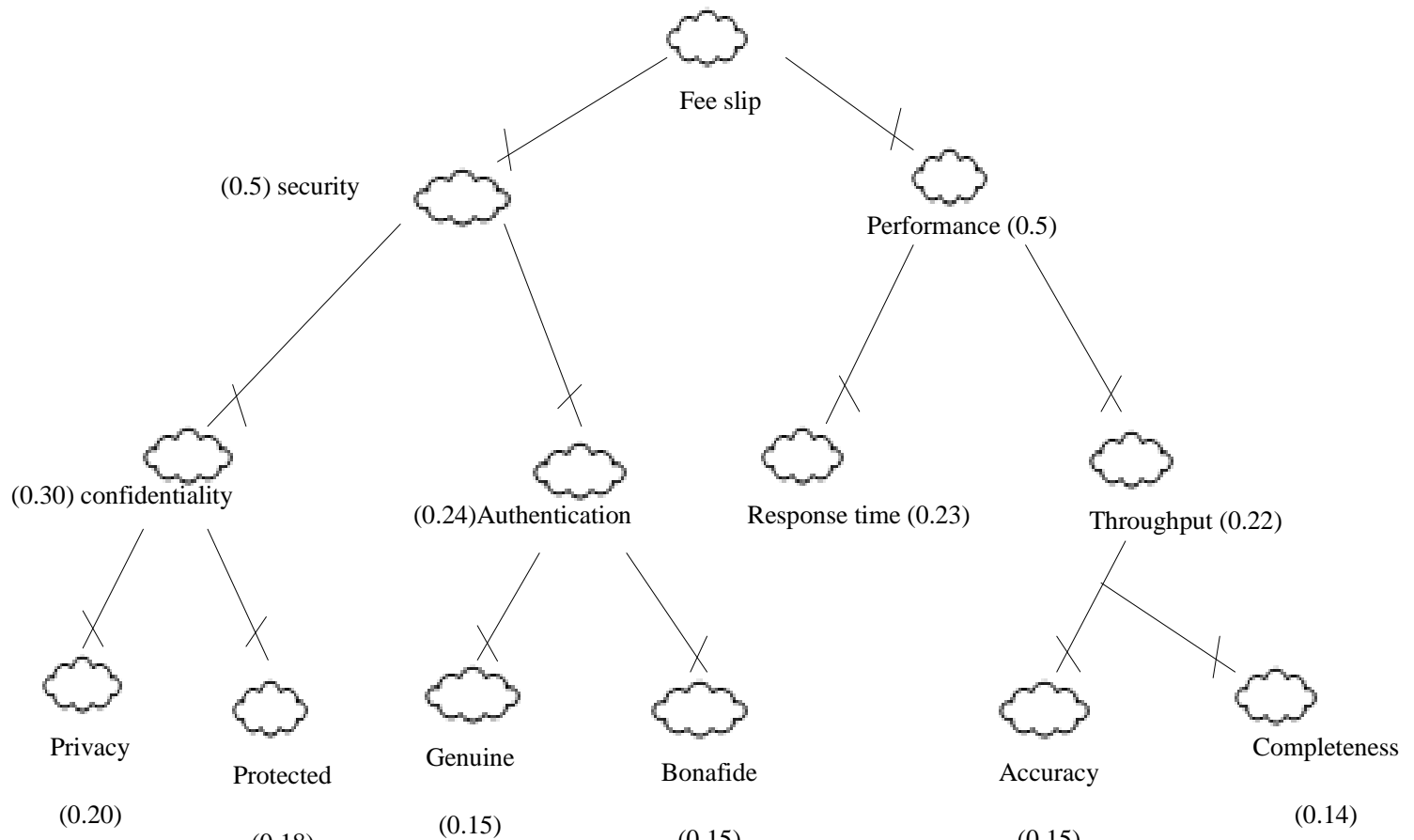

$(0.18)$

$(0.15)$

$(0.15)$

$(0.14)$

Fig. 3.2 .Normalized EFQQSIG

\section{CONCLUSION AND FUTURE WORK}

In this paper we present a method for prioritization of NFR using fuzzy linguistic variables when five decision maker involve in this process . The proposed method includes five step that is identifying stakeholders, decomposition of NFRs, collect experts fuzzy assessments, applying $\mathrm{L}^{-1}-\mathrm{R}^{-1}$ inverse function arithmetic principle and graded mean integration for the eliciting of decision makers weight and construct FQQSIG graph. In our case study we assume that five decision makers are involved in NFRs weight prioritization method. Future work includes:

1. To apply the proposed method in "Institute Examination System (IES)".

2. To strengthen the goal oriented elicitation process using proposed method. 


\section{REFERENCES}

[1] C.G. Sen, H. Barach, S. Sen, H. Bashgil, An integrated decision support system dealing with qualitative and quantitative objectives of enterprise software selection, Expert Systems with Applications 36 (3) (2009) 52725283.

[2] G. Zayaraz, P. Thambidurai, M. Srinivasan, P. Rodrigues, Software quality assurance through COSMIC FFP, ACM SIGSOFT Software Engineering Notes 30 (5) (2005)

[3] Gruia-Catalin Roman, A taxonomy of current issues in requirements engineering, IEEE Computer 18 (4) (1985) $14-23$

[4] H.W Chen, Ji Wang, W. Dong, High confidence Software technologies, Chinese journal of electronic 12 (2003) 1933-1938

[5] H.Y. Lin, P.Y. Hsu, G.J. Sheen, A fuzzy-based decisionmaking procedure for data warehouse system selection, Expert Systems with Applications 32 (3) (2007) 939-953

[6] ISO/IEC Standards 9126. International Standards Organization. Information technology-software product evaluation-quality characteristics and guidelines for their use, 1991

[7] L. Chung, B. Nixon, Dealing with nonfunctional requirements: three experimental studies of a processoriented approach, in: Proceedings of the $17^{\text {th }}$ International Conference on Software Engineering, (1995) 24-28.

[8] L. Chung, B. Nixon, E. Yu, J. Mylopoulos, NonFunctional Requirements in Software Engineering, Kluwer Academic, 2000.

[9] L. Chung, B. Nixon, et al, Non-functional Requirements in Software Engineering, Kluwer Academic Publishers, 1999

[10] L.A. Zadeh, Fuzzy set, Information and Control 8 (1965) 338-353

[11] L.A. Zadeh, Is there a need for fuzzy logic?, Information Sciences 178 (13) (2008) 2751-2779

[12] L.A. Zadeh, Toward a generalized theory of uncertainty (GTU) - an outline, Information Sciences 172 (2005) 140.

[13] M. Glinz, Rethinking the notion of non-functional requirements, in: Proceedings of the 3rd world congress for software quality, 2005, pp. 55-64.

[14] Mairiza Dewi and Zowghi Didar, "Constructing a Catalogue of conflicts among Non-functional requirements," L.A Maciaszek and P. Loucopoulos (Eds.):ENASE 2010, CCIS 230, pp.31-44, SpringerVerlag Berlin Heidelberg 2011.
[15] Ming-Xun Zhu Xin-Xing Luo, Xiao-Hong chen, Desheng Dash Wu "A non-functional requirements tradeoff model in Trustworthy software" Elsevier.

[16] Nida Afreen, Asma Khatoon, and Mohd. Sadiq, " A Taxonomy of Software's Non-Functional Requirements", $2^{\text {nd }}$ International Conference on Computer and Communication Technologies (IC3T), Springer, July, 2015, Hyderabad, India (Accepted for Publication).

[17] Sadiq Mohd,Jain S.K "Applying fuzzy preference relation for requirement prioritization in goal oriented Requirement" International Journal of system assurance engineering and management ,Springer Vol.5 issue 4 pp.711-723,2014

[18] Sadiq M and Jain SK, "A Fuzzy Based Approach for Requirements Prioritization in Goal Oriented Requirements Elicitation Process," International Conference of Software Engineering and Knowledge Engineering (SEKE), pp. 54-58, 2013.

[19] Sadiq M and Jain SK, "Stakeholders Identification Methods in Goal Oriented Requirements Elicitation Process," Fifth IEEE International Workshop on Requirements Prioritization and Communication at IEEE International Requirements Engineering Conference (RE), pp. 25-33, 2014 Karlskrona, Sweden.

[20] T. Marew et al Tactics based approach for integrating non- functional requirements in object oriented analysis and design, Syst. Software 82 (2009)1642-1656.

[21] V. Sadana X.liu Analysis of conflict among nonfunctional requirement using integrated analysis of functional and non-functional requirements computer software and applications conference, COMPSAC 2007.

[22] X.B. Illa, X. Franch, J.A. Pastor, Formalising ERP selection criteria, in: Proceedings of the 10th international workshop on software specification and design (IWSSD'00), Shelter Island, San Diego, California, 2000, pp. 115-123.

[23] Y. Peng, G. Wang, H. Wang, User preferences based software defect detection algorithms selection using MCDM, Information Sciences 191 (2012) 3-13.

[24] Z-p. Fan, Y. Liu, A Method for Group Decision-making based on Multi-granularity uncertain linguistic information, Expert systems with Applications 37 (2010) 4000-4008.

[25] Sommerville ,I.:Software Engineering, $7^{\text {th }}$ Edn.Pearson Education limited,Essex(2004).

[26] Boehm,B.,In,H.:Identifying Quality -Requirements Conflict.IEEE Software 13,25-35(1996).

[27] Cho C.C., "The Canonical Representation of Multiplication Operation on Triangular Fuzzy Numbers", The international Journal Computers and Mathematics with Application, PP.1601-1610.2003. 